\title{
Estudo epidemiológico descritivo do carcinoma epidermóide bucal em uma população brasileira
}

\section{Descriptive epidemiological study of oral squamous cell carcinoma in a brazilian population}

\author{
Fernando Augusto Cervantes Garcia de SOUSA \\ Thaís Cachuté PARADELLA \\ Doutorando - Programa de Pós-Graduação em Biopatologia Bucal - Área de Concentração Biopatologia Bucal - \\ Faculdade de Odontologia de São José dos Campos - UNESP - Universidade Estadual Paulista - São José dos Campos \\ $-\mathrm{SP}-$ Brasil
}

\section{Luiz Eduardo Blumer ROSA}

Professor Adjunto - Disciplina de Patologia Bucal - Departamento de Biociências e Diagnóstico Bucal - Faculdade de Odontologia de São José dos Campos - UNESP - Universidade Estadual Paulista - São José dos Campos - SP - Brasil

\section{Horácio FAIG LEITE}

Professor Titular - Disciplina de Anatomia - Departamento de Biociências e Diagnóstico Bucal - Faculdade de Odontologia de São José dos Campos - UNESP - Universidade Estadual Paulista - São José dos Campos - SP - Brasil

\begin{abstract}
Resumo
O objetivo deste trabalho é descrever as características epidemiológicas dos casos de carcinoma epidermóide numa população brasileira. Foram analisados os laudos com o diagnóstico histopatológico de carcinoma epidermóide em mucosa bucal emitidos por um importante centro de referência em Patologia Bucal da cidade de São José dos Campos - SP entre janeiro de 1972 e outubro de 2007. Dos 271 casos diagnosticados, 64,45\% acometeram indivíduos do gênero masculino e da raça branca. Quanto à idade, $88,76 \%$ dos casos ocorreram entre a quinta e a oitava década de vida, sendo a média de idade no momento do diagnóstico para o gênero masculino de 58,79 $\pm 12,47$ anos e para o gênero feminino de 63,94 \pm 13,46 anos. Em cerca de 10\% dos casos foi relatado tabagismo e/ou alcoolismo. A língua foi a região mais freqüentemente acometida (23,17\%), seguida pelo lábio $(22,01 \%)$ e pelo assoalho bucal $(15,06 \%)$. Clinicamente, o tamanho da lesão primária no momento do diagnóstico foi, em média, 2,26 $\pm 1,17 \mathrm{~cm}$ e seu tempo de evolução foi 9,13 $\pm 11,75$ meses para o gênero masculino e $6,46 \pm 8,83$ meses para o gênero feminino. Em 37,64\% dos casos foi relatada dor. Em 90,41\% dos casos diagnosticados houve concordância entre o diagnóstico clinico e o histopatológico. Quanto ao grau de diferenciação histológica, 38,89\% dos casos diagnosticados eram bem diferenciados, 50,00\% moderadamente diferenciados e $11,11 \%$ pouco diferenciados. Estes resultados estão de acordo com a literatura e reforçam a importância de políticas públicas de combate ao câncer bucal.
\end{abstract}

\section{UNITERMOS}

Câncer bucal; carcinoma epidermóide; mucosa bucal; epidemiologia.

\section{INTRODUÇÃO}

O câncer bucal é um problema de saúde pública em muitos paises ${ }^{1,5-8,14-15,18,20,22,24}$, inclusive no Brasil ${ }^{2,9,12}$. Segundo estimativas do Instituto Nacional do Câncer (INCA) para o ano de 2006, foram diagnosticados no país 472.050 novos casos de câncer, sendo que, destes, 13.470 acometeram a cavidade bucal, colocando-a como o sexto local de maior incidência da doença entre os homens e o oitavo entre as mulheres ${ }^{2}$. Independentemente do gênero, a maioria dos casos diagnosticados corresponde ao carcinoma epidermóide $\mathrm{e}$ está relacionada a fatores de risco como tabagismo, alcoolismo, hábitos alimentares, fatores ocupacionais, radiação solar, entre outros 5,6,8-10,15-14,18,20-21,24. 
Todavia, apesar dos fatores de risco serem bem conhecidos, favorecendo sua prevenção, estima-se que somente em 2005 o câncer bucal foi responsável por 5.818 óbitos, ou seja, aproximadamente $4 \%$ das mortes causadas pelo câncer naquele ano ${ }^{3}$. Além disso, a maioria dos casos ainda é diagnosticada tardiamente, implicando mutilações, que, muitas vezes, inabilitam o indivíduo para as integrações familiares, sociais e profissionais, temporária ou definitivamente ${ }^{1,9,13,16,22}$.

Assim, o objetivo deste trabalho é descrever as características epidemiológicas dos casos de carcinoma epidermóide diagnosticados na cidade de São José dos Campos - SP entre 1972 e 2007 e compará-las com a literatura, contribuindo para o estabelecimento de políticas públicas de prevenção, diagnóstico e tratamento do câncer bucal.

\section{Material e Método}

Foram avaliados todos os laudos com o diagnóstico histopatológico de carcinoma epidermóide em mucosa bucal emitidos por um importante centro de referência em Patologia Bucal da cidade de São José dos Campos - SP entre janeiro de 1972 e outubro de 2007. A seguir, foram selecionados os dados referentes ao gênero, raça, idade, hábitos, localização da lesão primária, tamanho, tempo de evolução, sintomatologia, diagnóstico clínico e grau de diferenciação histológica. Os dados foram então tabulados e analisados descritivamente.

\section{Resultados}

Entre janeiro de 1972 e outubro de 2007, foram diagnosticados 271 casos de carcinoma epidermóide em mucosa bucal, com uma média de 7,53 casos por ano. No entanto, observa-se que, a partir de 1993, esta média foi superior a 15, mostrando um aumento considerável em relação ao período de 1972 a 1992, cuja média mantevese inferior a três casos por ano (Gráfico 1).

Dos 271 casos diagnosticados, 64,45\% acometeram indivíduos do gênero masculino e da raça branca. Quanto à idade, 88,76\% dos casos ocorreram entre a quinta e a oitava década de vida, sendo a média de idade no momento do diagnóstico para o gênero masculino de $58,79 \pm 12,47$ anos e para o gênero feminino de 63,94 \pm 13,46 anos (Gráfico 2). Em cerca de 10\% dos casos foi relatado tabagismo e/ou alcoolismo.

A língua foi a região mais freqüentemente acometida $(23,17 \%)$, seguida pelo lábio $(22,01 \%)$ e pelo assoalho bucal $(15,06 \%)$ (Gráfico 3). Clinicamente, o tamanho da lesão primária no momento do diagnóstico foi, em média, 2,26 $\pm 1,17 \mathrm{~cm}$ e seu tempo de evolução foi $9,13 \pm 11,75$ meses para o gênero masculino e $6,46 \pm 8,83$ meses para o gênero feminino. Em $37,64 \%$ dos casos foi relatada dor.

Em 90,41\% dos casos diagnosticados houve concordância entre o diagnóstico clinico e o histopatológico. Quanto ao grau de diferenciação histológica, 38,89\% dos casos diagnosticados eram bem diferenciados, 50,00\% moderadamente diferenciados e $11,11 \%$ pouco diferenciados.

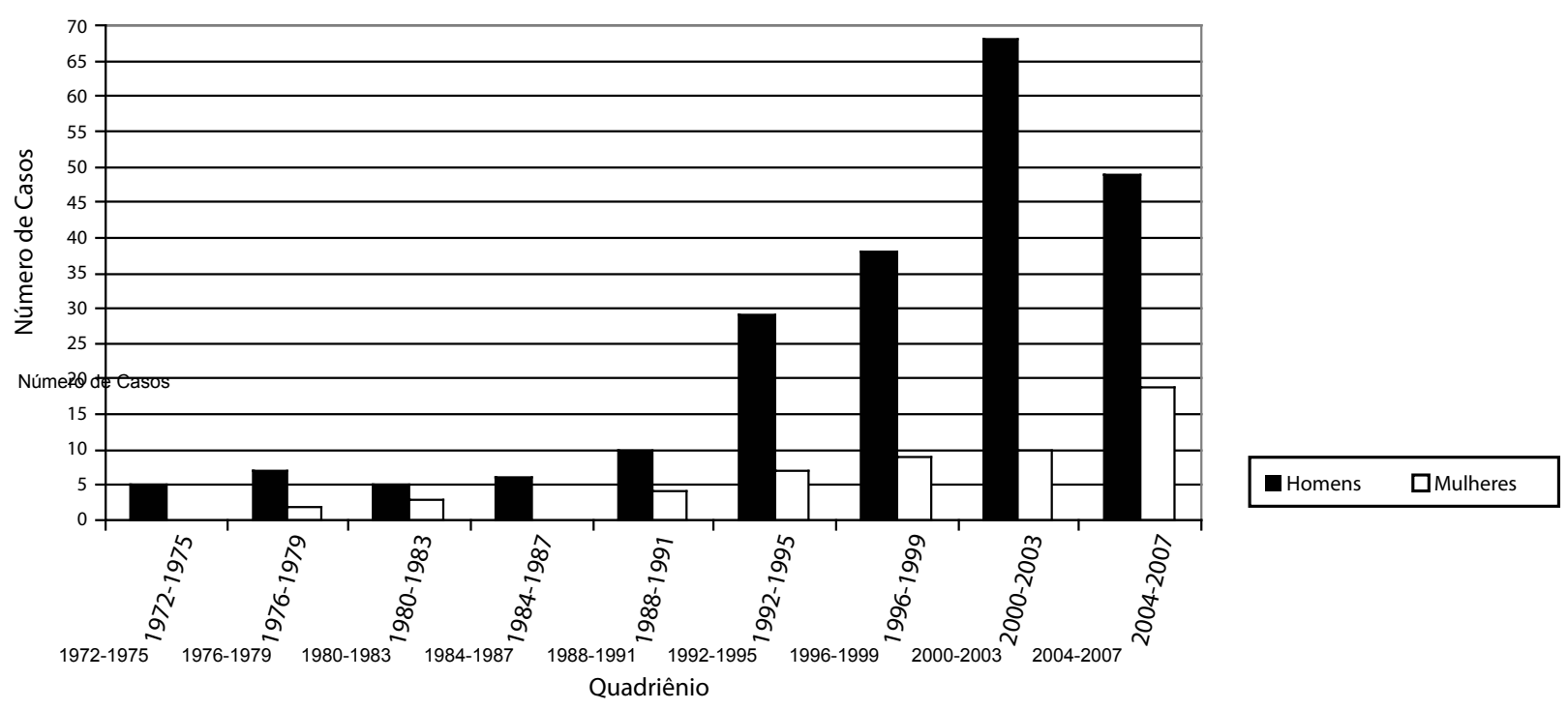

Gráfico 1 - Distribuição do número de casos diagnosticado por quadriênio. 
Sousa FACG, Paradella TC, Rosa LEB, Faig Leite H

ESTUDO EPIDEMIOLÓGICO DESCRITIVO DO CARCINOMA EPIDERMÓIDE BUCAL EM UMA POPULAÇÃO BRASILEIRA

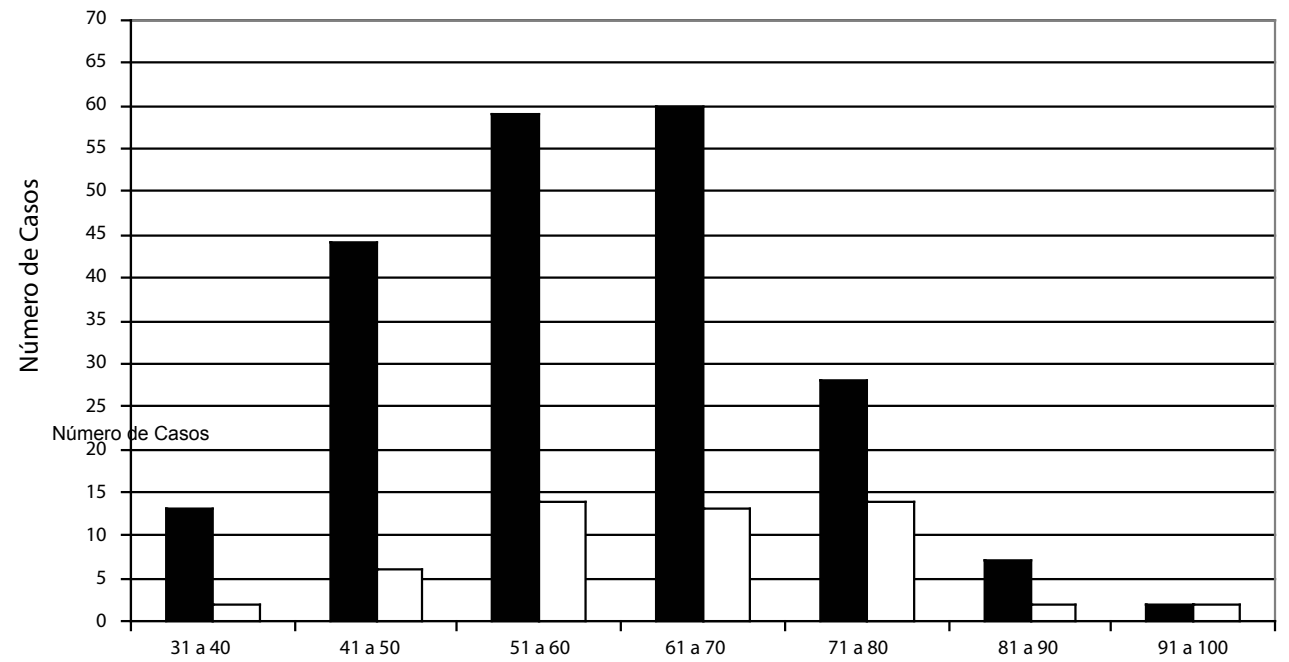

Faixa Etária

Domens $\square$ Mulheres

Gráfico 2 - Distribuição do número de casos diagnosticados por faixa etária.



Localização

Domens $\square$ Mulheres

Gráfico 3 - Número de casos diagnosticados de acordo com a localização da lesão primária. 


\section{Dıscussão}

O número de casos de câncer bucal vem crescendo no Brasil e no mundo, especialmente entre as mulheres. ${ }^{7,9,18,20,22}$. O aumento da incidência de câncer bucal pode estar relacionado à maior exposição da população mundial a fatores de risco como tabagismo, consumo excessivo de álcool e exposição solar ${ }^{7,18,20}$ e à maior procura por atendimento médico-odontológico especializado, favorecendo o diagnóstico da doença.

Os homens são mais acometidos pelo câncer bucal que as mulheres, numa razão média de 1,85:1, todavia a razão entre homens e mulheres pode variar de acordo com o país e a faixa etária analisados e vem diminuindo nos últimos anos ${ }^{1,6,7,9,14,20}$. Ao contrário do gênero, a raça não é fator um determinante para o câncer bucal, embora tenha sido observada no Brasil uma maior predileção da doença pela raça branca9.

A maioria dos casos de câncer bucal é diagnosticada em indivíduos com mais de 50 anos $^{4,6,8,9,14,20,22}$. Os homens são acometidos mais precocemente que as mulheres ${ }^{6,14,20}$. Isto, provavelmente, porque no gênero masculino o consumo de tabaco e/ou álcool começa mais cedo. Realmente, diversos estudos comprovam que a maioria dos casos de câncer bucal pode ser atribuída ao consumo de tabaco e/ ou álcool ${ }^{5-6,8-10,14-15,18,20-21,24}$.

A borda lateral da língua, seguida pelo assoalho bucal, é a localização de maior prevalência do carcinoma epidermóide ${ }^{1,5,9,20}$, possivelmente, por causa da conformação anatômica da cavidade bucal, que aumenta o tempo de contato entre estas estruturas e substâncias carcinógenas diluídas na saliva, especialmente, em indivíduos fumantes ${ }^{6}$. Outra causa importante parece estar relacionada com o fato destas áreas apresentarem regiões de mucosas compostas por epitélio de transição, o que parece favorecer o aparecimento de alterações genéticas com mais freqüência do que em áreas de mucosa de revestimento ou especializada. Outra localização de alta prevalência do carcinoma epidermóide é o lábio inferior, todavia, neste, a exposição à radiação solar parece ser o principal fator de risco ${ }^{6,8,9,20}$.

Apesar da cavidade bucal favorecer o exame clínico, grande parte dos casos de câncer bucal é diagnóstica em estágios avançados ${ }^{1,9}$, tornando o prognóstico da doença bastante desfavorável l, $^{1,13,16,22}$.
Lesões diagnosticadas com mais de $5 \mathrm{~mm}$ de espessura tem alta probabilidade de apresentar metástase oculta em linfonodos cervicais ${ }^{17}$. O aparecimento de metástases influência fortemente prognóstico, pois aumenta significantemente as chances de recorrência e reduz a sobrevida global ${ }^{11,16,19,23}$. O atraso no diagnóstico do câncer bucal se deve principalmente ao longo período de espera antes do individuo com a doença procurar por atendimento médico-odontológico. A ausência de sintomatologia nos estágios iniciais do carcinoma epidermóide também favorece o atraso no seu diagnóstico. Em menos de um quarto dos casos, a dor é relatada nos estágios iniciais (T1 ou T2 e N0) ${ }^{22}$.

Todavia, as características clínicas do carcinoma epidermóide em mucosa bucal são bem definidas, favorecendo o diagnóstico correto da doença. Além disso, a maioria das lesões diagnosticadas é bem diferenciada ${ }^{6,8}$, compensando parcialmente a demora no seu diagnóstico, uma vez que lesões bem diferenciadas apresentam crescimento mais lento que lesões moderadamente ou pouco diferenciadas, embora a relação entre o grau de diferenciação histológica e o prognóstico da doença ainda hoje é alvo de muita controvérsia ${ }^{16}$.

Por fim, o quadro 1 mostra resumidamente os resultados de alguns levantamentos epidemiológicos publicados recentemente na literatura internacional sobre o câncer bucal, permitindo compará-los com os resultados obtidos neste trabalho. Comparação esta que evidencia a concordância entre os nossos resultados e os da literatura (Quadro 1).

\section{Conclusão}

Os resultados deste estudo estão de acordo com a literatura, sugerindo que o câncer bucal no Brasil segue as tendências observadas em outros países. Além disso, este estudo reforça a necessidade de se estabelecer políticas públicas de combate ao câncer bucal, enfatizando, principalmente, a importância da prevenção e do diagnóstico precoce da doença. Infelizmente, a falta de informação e o preconceito fazem com que os indivíduos acometidos pelo câncer bucal esperem por meses antes de procurar por atendimento médico-odontológico adequado, aumentado consideravelmente os danos causados pela doença e reduzindo sua expectativa de vida. 


\section{Quadro 1 - Resultados de alguns levantamentos epidemiológicos publicados recentemente na literatura interna- cional sobre o câncer bucal.}

\begin{tabular}{|c|c|c|c|c|c|c|}
\hline Autores & País & $\begin{array}{c}\text { Gênero } \\
\text { (H:M) }\end{array}$ & Raça & Idade & Hábitos & Localização \\
\hline $\begin{array}{c}\text { Brandizzi et al. }{ }^{1} \\
(2005)\end{array}$ & Argentina & $1,2: 1$ & Branca & --- & -- & Língua \\
\hline $\begin{array}{c}\text { Bhurgri et al. }{ }^{4} \\
(2004)\end{array}$ & Paquistão & $1,7: 1$ & Branca & $6^{a}$ década & --- & --- \\
\hline $\begin{array}{c}\text { Canto \& Devesa }{ }^{5} \\
(2002)\end{array}$ & Estados Unidos & $2,4: 1$ & Branca & --- & --- & Língua \\
\hline $\begin{array}{c}\text { Chidzonga \& } \\
\text { Mahomva }^{6}(2006)\end{array}$ & Zimbábue & $2,0: 1$ & Negra & $5^{a}$ e $6^{a}$ décadas & --- & Gengiva \\
\hline $\begin{array}{c}\text { Conway et al. }{ }^{7} \\
(2006)\end{array}$ & Reino Unido & $1,8: 1$ & Branca & $6^{a}$ e $7^{a}$ décadas & $\begin{array}{c}\text { Tabagismo e/ou } \\
\text { alcoolismo }\end{array}$ & --- \\
\hline $\begin{array}{c}\text { De Visscher et al. }{ }^{8} \\
(1998)\end{array}$ & Holanda & $5,7: 1$ & Branca & $7^{a}$ e $8^{a}$ décadas & --- & Lábio* \\
\hline $\begin{array}{c}\text { Durazzo et al. }{ }^{9} \\
\text { (2005) }\end{array}$ & Brasil & $2,1: 1$ & Branca & $6^{a}$ década & $\begin{array}{l}\text { Tabagismo e/ou } \\
\text { alcoolismo }\end{array}$ & $\begin{array}{c}\text { Língua e/ } \\
\text { ou assoalho } \\
\text { bucal }\end{array}$ \\
\hline $\begin{array}{c}\text { Gervásio et al. }{ }^{12} \\
(2001)\end{array}$ & Brasil & $4,8: 1$ & Branca & $6^{a}$ década & $\begin{array}{c}\text { Tabagismo e/ou } \\
\text { alcoolismo }\end{array}$ & Língua \\
\hline Lam et al. ${ }^{14}$ (2006) & Austália & 1,9:1 & Branca & $7^{a}$ década & $-\cdots$ & Língua** \\
\hline $\begin{array}{l}\text { Llewellyn et al. }{ }^{15} \\
(2003)\end{array}$ & Reino Unido & 1,3:1 & Branca & $5^{a}$ década ${ }^{\star \star \star}$ & $\begin{array}{c}\text { Tabagismo e/ou } \\
\text { alcoolismo }\end{array}$ & Língua \\
\hline $\begin{array}{c}\text { Tarvainen et al. }{ }^{20} \\
(2004)\end{array}$ & Finlândia & $2,0: 1$ & Branca & $7^{a}$ década & $\begin{array}{c}\text { Tabagismo e/ou } \\
\text { alcoolismo }\end{array}$ & Lábio \\
\hline
\end{tabular}

*foram incluídos apenas pacientes com lesão em lábio; **foram incluídos apenas pacientes com lesão em língua; ***foram incluídos apenas pacientes com 45 anos ou menos.

\section{Abstract}

The purpose of this study was to describe the epidemiological characteristics of the cases of oral squamous cell carcinoma in a Brazilian population. Examination charts with the diagnosis of oral squamous cell carcinoma issued by an Oral Pathology reference center were analyzed. Two hundred and seventy-one cases were included in the study, being $64.45 \%$ of male Caucasian individuals. Regarding age, $88.76 \%$ of the cases occurred between the fifth and eighth decade of life, being the mean age $58.79 \pm 12.47$ years for men and $63.94 \pm 13.46$ years for women. The tongue was the most frequent affected site $(23.17 \%)$, followed by lip $(22.01 \%)$ and floor of mouth $(15.06 \%)$. Clinically, the mean size of the primary lesion at the moment of the diagnosis was $2.26 \pm 1.17 \mathrm{~cm}$ and its mean evolution time was $9.13 \pm 11.75$ months for men and $6.46 \pm 8.83$ months for women. Regarding the histological degree of differentiation, $38.89 \%$ of the diagnosed cases were well-differentiated, $50 \%$ moderately differentiated and $11.11 \%$ poorly differentiated. These results are in agreement with the findings of the literature and reinforce the importance of public programs of oral cancer prevention.

\section{UNITERNS}

Oral cance; squamous cell carcinoma; mouth mucos; epidemiology.

\section{REFERÊNCIAS}

1. Brandizzi D, Chuchurru JA, Lanfranchi HE, Cabrini RL. Analysis of the epidemiological features of oral cancer in the city of Buenos Aires. Acta Odontol Latinoam. 2005;18(1):31-5.
2. Brasil. Ministério da Saúde. Instituto Nacional do Câncer. Estimativa 2006: incidência de câncer no Brasil. Rio de Janeiro: INCA; 2005. 94 p.

3. Brasil. Ministério da Saúde. Secretária de Vigilância em Saúde. Sistema de informações sobre mortalidade (SIM) [database on the Internet]. Brasília: Secretária de Vigilância em Saúde [cited 2007 Nov 13]. Available from: http://tabnet.datasus.gov.br/cgi/deftohtm.exe?sim/ cnv/obtuf.def 
4. Bhurgri Y, Bhurgri A, Usman A, Pervez S, Kayani N, Bashir I, et al. Epidemiological review of head and neck cancers in Karachi. Asian Pac J Cancer Prev. 2006;7(2):195-200

5. Canto MT, Devesa SS. Oral cavity and pharynx cancer incidence rates in the United States, 1975-1998. Oral Oncol. 2002;38(6):610-7.

6. Chidzonga MM, Mahomva L. Squamous cell carcinoma of the oral cavity, maxillary antrum and lip in a Zimbabwean population: a descriptive epidemiological study. Oral Oncol. 2006;42(2):184-9.

7. Conway DI, Stockton DL, Warnakulasuriya KA, Ogden G, Macpherson LM. Incidence of oral and oropharyngeal cancer in United Kingdom (1990-1999) - recent trends and regional variation. Oral Oncol. 2006;42(6):586-92.

8. De Visscher JG, Schaapveld M, Otter R, Visser O, van der Waal I. Epidemiology of cancer of the lip in The Netherlands. Oral Oncol. 1998;34(5):421-6.

9. Durazzo MD, de Araújo CE, Brandão Neto J de S, Potenza A de S, Costa P, Takeda F, et al. Clinical and epidemiological features of oral cancer in a medical school teaching hospital from 1994 to 2002: increasing incidence in women, predominance of advanced local disease, and low incidence of neck metastases. Clinics. 2005;60(4):293-8.

10. Franco EL, Kowalski LP, Oliveira BV, Curado MP, Pereira RN, Silva $\mathrm{ME}$, et al. Risk factors for oral cancer in Brazil: a case-control study. Int J Cancer. 1989;43(6):992-1000.

11. Genden EM, Ferlito A, Bradley PJ, Rinaldo A, Scully C. Neck disease and distant metastases. Oral Oncol. 2003;39(3):207-12.

12. Gervásio OL, Dutra RA, Tartaglia SM, Vasconcellos WA, Barbosa AA, Aguiar MC. Oral squamous cell carcinoma: a retrospective study of 740 cases in a Brazilian population. Braz Dent J. 2001;12(1):57-61.

13. Kowalski LP, Franco EL, Torloni H, Fava AS, de Andrade Sobrinho $\mathrm{J}$, Ramos G, et al. Lateness of diagnosis of oral and oropharyngeal carcinoma: factors related to the tumour, the patient and health professionals. Eur J Cancer B Oral Oncol. 1994;30B(3):167-73.

14. Lam L, Logan RM, Luke C. Epidemiological analysis of tongue cancer in South Australia for the 24-year period, 1977-2001. Aust Dent J. 2006;51(1):16-22.

15. Llewellyn CD, Linklater K, Bell J, Johnson NW, Warnakulasuriya KA. Squamous cell carcinoma of the oral cavity in patients aged 45 years and under: a descriptive analysis of 116 cases diagnosed in the South East of England from 1990 to 1997. Oral Oncol. 2003;39(2):106-14.
16. Massano J, Regateiro FS, Januário G, Ferreira A. Oral squamous cell carcinoma: review of prognostic and predictive factors. Oral Surg Oral Med Oral Pathol Oral Radiol Endod. 2006;102(1):67-76.

17. O-charoenrat P, Pillai G, Patel S, Fisher C, Archer D, Eccles S, et al. Tumour thickness predicts cervical nodal metastases and survival in early oral tongue cancer. Oral Oncol. 2003;39(4):386-90.

18. Sarachev EL, Ananostev NH. Tendencies in the oral cavity cancer morbidity in three regions of South Bulgaria for a period of 15 years (1985-1999). Folia Med (Plovdiv). 2001;43(1-2):150-4.

19. Shingaki S, Takada M, Sasai K, Bibi R, Kobayashi T, Nomura T, et al. Impact of lymph node metastasis on the pattern of failure and survival in oral carcinomas. Am J Surg. 2003;185(3):278-84.

20. Tarvainen L, Suuronen R, Lindqvist C, Malila N. Is the incidence of oral and pharyngeal cancer increasing in Finland? An epidemiological study of 17,383 cases in 1953-1999. Oral Dis. 2004;10(3):167-72.

21. Thomas G, Hashibe M, Jacob BJ, Ramadas K, Mathew B, Sankaranarayanan $\mathrm{R}$, et al. Risk factors for multiple oral premalignant lesions. Int J Cancer. 2003;107(2):285-91.

22. Tromp DM, Brouha XD, Hordijk GJ, Winnubst JA, de Leeuw RJ. Patient and tumour factors associated with advanced carcinomas of the head and neck. Oral Oncol. 2005;41(3):313-9.

23. Woolgar JA, Rogers SN, Lowe D, Brown JS, Vaughan ED. Cervical lymph node metastasis in oral cancer: the importance of even microscopic extracapsular spread. Oral Oncol. 2003;39(2):130-7.

24. Yang Y-H, Chen C-H, Chang JSF, Lin C-C., Cheng T-C, Shieh, T-Y. Incidence rates of oral cancer and oral pre-cancercous lesions in a 6-year follow-up study of a Taiwanese aboriginal community. J Oral Pathol Med. 2005;34(10):596-601.

Recebido em 18/02/08 Aprovado em 12/06/08

Correspondência:

Fernando Augusto Cervantes Garcia de Sousa Rua Irmã Maria Demétria Kfuri, 196 - Jardim Esplanada II CEP 12242-500 São José dos Campos - SP - Brasil E-mail: facgs@uol.com.br 